\title{
El estudio de caso en la investigación de las ecologías de aprendizaje del docente universitario
}

\author{
Iris Estévez, Alba Souto-Seijo y Mercedes González-Sanmamed \\ Universidade da Coruña, España | iris.estevezb@udc.es| \\ https://orcid.org/0000-0003-2821-5663 | \\ a.souto1@udc.es |https://orcid.org/0000-0002-9140-3184 | \\ mercedes.gonzalez.sanmamed@udc.es | https://orcid.org/0000-0002-3410-6810
}

\begin{abstract}
Resumen: El concepto de Ecologías de Aprendizaje nos proporciona un marco útil para explicar cómo tiene lugar el aprendizaje en la actualidad. El presente trabajo responde a dos objetivos principales: a) evidenciar la pertinencia del Estudio de Caso como diseño de investigación para abordar el estudio de las Ecologías de Aprendizaje, y b) analizar cómo los mejores docentes universitarios españoles configuran sus Ecologías de Aprendizaje y las utilizan en beneficio de su desarrollo profesional. Así pues, se ha seleccionado la tradición de Estudio de Caso para el desarrollo de la investigación. Se analizaron 72 casos de docentes excelentes a partir de la realización de dos entrevistas en profundidad. Los resultados han permitido identificar dos grandes dimensiones en el análisis de las Ecologías de Aprendizaje: una personal (motivación, identidad profesional y concepciones) y otra contextual (relaciones, actividades y recursos tecnológicos). A partir de la consideración de estos elementos se puede esbozar un panorama de cuáles pueden ser los factores determinantes de la excelencia docente dentro del contexto universitario. Asimismo, los resultados muestran las ventajas del enfoque cualitativo, específicamente del Estudio de Caso, en el análisis de realidades tan complejas como la que subyace al fenómeno objeto de estudio.
\end{abstract}

Palabras Clave: Ecologías de Aprendizaje; Docencia; Universidad; Investigación Cualitativa; Estudio de Caso.

\section{Introducción}

El Equipo de Investigación Educativa (EIRA) de la Universidade da Coruña tiene como principales líneas de investigación:

a) la innovación y mejora de la enseñanza universitaria;

b) los procesos de formación para el desarrollo organizativo y profesional; y

c) la innovación a través de la integración de la tecnología en la educación.

Actualmente, el Equipo está centrado en la investigación sobre las Ecologías de Aprendizaje (EdA), un concepto emergente que está despertando un gran interés en el ámbito educativo y en la comunidad investigadora. Prueba de ello son los diferentes estudios y proyectos de investigación nacionales e internacionales que se están desarrollando para incrementar el conocimiento acerca de esta incipiente perspectiva. De hecho, esta investigación se enmarca dentro del Proyecto "Cómo aprenden los mejores Docentes Universitarios en la Era Digital: Impacto de las Ecologías de Aprendizaje en la Calidad de la Enseñanza" (ECO4LEARN-HE), financiado por el Ministerio de Economía y Finanzas (Referencia EDU2015-67907-R) y en el que colaboran 20 investigadores de un total de seis universidades españolas.

En base a todo lo anteriormente descrito, el presente trabajo tiene dos principales objetivos. Por un lado, analizar cómo los mejores docentes universitarios españoles configuran sus Ecologías de Aprendizaje y las utilizan en beneficio de su desarrollo profesional y, en segundo lugar, evidenciar la pertinencia del Estudio de Caso como diseño de investigación para abordar el análisis de las Ecologías de Aprendizaje.

Las EdA se caracterizan por ser un constructo complejo y multidimensional, por lo que la metodología cualitativa se convierte en la opción más adecuada para abordar su estudio. 
Concretamente, en el presente trabajo se fundamenta la pertinencia del Estudio de Caso para analizar las EdA de una muestra de los mejores docentes universitarios de España. Para ello se describirá detalladamente el estudio empírico desarrollado.

En primer lugar, se exponen las fases a través de las cuales se ha llevado a cabo la investigación y, a continuación, se explica el proceso de diseño y realización de la técnica de recogida de datos seleccionada, la entrevista. En tercer lugar, se describen los participantes del estudio y, finalmente, se especifica cómo se ha realizado el análisis de los datos y se comentan algunos resultados. Seguidamente, se muestran una serie de recomendaciones para futuras investigaciones que aborden el estudio del aprendizaje desde una perspectiva ecológica. Asimismo, en el apartado denominado Aplicabilidad en otros contextos, se exponen las implicaciones prácticas de este trabajo. Finalmente, se presentan las conclusiones generales emanadas de los resultados del estudio.

\subsection{Las Ecologías de Aprendizaje desde una perspectiva cualitativa}

Nos encontramos inmersos en la Sociedad de la Información y del Conocimiento (SIC), denominada así por el importante papel que juegan ambos elementos (FernándezEnguita, 2016). Una sociedad que se caracteriza por la presencia de la tecnología en todos los ámbitos de la vida. Entre otras cosas, la tecnología ha deslocalizado el conocimiento y ha modificado la velocidad con la que este se genera y se transmite (Duart \& MengualAndrés, 2014; González-Sanmamed, Sangrà, Souto-Seijo, \& Estévez, 2018). Así, para poder hacer frente a esta permanente renovación del conocimiento, los ciudadanos de la SIC nos vemos obligados a aprender a lo largo y ancho de la vida (González-Sanmamed, Estévez, Souto-Seijo, \& Muñoz-Carril, 2020). Este aspecto cobra mayor importancia, si cabe, en el caso de los docentes, puesto que tienen la difícil tarea de enseñar en un mundo cambiante.

De acuerdo con González-Sanmamed y colaboradores (2018), la forma en que aprendemos se ha transformado profundamente, lo cual ha contribuido a que surjan nuevos conceptos para explicar cómo tiene lugar el aprendizaje en la SIC. Uno de estos conceptos es el de EdA, el cual nos proporciona un marco útil para explicar cómo aprendemos, y qué contextos y/o elementos empleamos para formarnos, con el fin de proporcionarnos nuevas oportunidades de aprendizaje.

A pesar de que este concepto está despertando un gran interés, las EdA se consideran todavía una línea de investigación emergente que se encuentra en estado embrionario dado el carácter complejo, multidimensional y poliédrico del constructo (GonzálezSanmamed, Muñoz-Carril, \& Santos-Caamaño, 2019). Barron (2004) establece que las EdA son "el conjunto de contextos hallados en espacios físicos o virtuales que proporcionan oportunidades de aprendizaje. Cada contexto comprende una configuración única de actividades, recursos materiales, relaciones personales y las interacciones que surgen de ellos" (p. 6). Esta es una de las definiciones más citadas en las diversas investigaciones sobre EdA. Sin embargo, en una reciente revisión sistemática, Sangrá, Raffaghelli y Guitert (2019) determinaron que a día de hoy no hay una definición clara del concepto, porque los estudios publicados pertenecen a diversas disciplinas.

Por todo ello, el estudio de este fenómeno nos obliga a adoptar una metodología de carácter cualitativo. Esta metodología es una de las más empleadas en la investigación en Ciencias Sociales por varios motivos, entre los que destacan: la posibilidad de abordar los fenómenos sociales desde una perspectiva holística, la consideración de las características del objeto de estudio de forma integrada y articulada o la riqueza informativa de los datos obtenidos (Verd \& Lozares, 2016). De acuerdo con Pedraz, Zarco, Ramasco, y Palmar (2014), la investigación cualitativa "es esencial para la comprensión de realidades complejas y diversas, para explorar los significados de la experiencia humana y aproximarse a ellos, y para captar los elementos subjetivos contextuales de los procesos sociales" (p. 9). Este tipo de investigación nos permitirá comprender e interpretar la realidad con la finalidad de construir nuevo conocimiento (Arantzamendi, LópezDicastillo, \& García-Vivar, 2012) y, por ese motivo, se ha considerado la óptica más adecuada. 
Finalmente, y antes de explicar la tradición de investigación de Estudio de Caso adoptada, quisiéramos destacar la importancia de seguir investigando sobre el tema de las Ecologías de Aprendizaje, puesto que nos permitirá comprender cada vez más y mejor cómo pueden aprovecharse todos los recursos y oportunidades de aprendizaje que los individuos tienen en un contexto social cada vez más difuso a causa de la acción de las tecnologías digitales. Tal y como afirman González-Sanmamed y colaboradores (2018) y Sangrà, GonzálezSanmamed y Guitert-Catasús (2013), las EdA se muestran como una estrategia adecuada que contribuye al desarrollo profesional de los docentes, dado que les ayuda a actualizarse de forma autodirigida y eficaz. Así, se puede afirmar que la investigación sobre EdA impactará en la práctica y mejorará las vías de desarrollo profesional y, por tanto, la calidad de la enseñanza, a nivel individual, colectivo e institucional.

\subsection{La pertinencia del Estudio de Caso en el escenario ecológico}

Se ha optado por el diseño de Estudio de Caso para implementar y dar sentido a este proyecto, porque esta es una tradición de investigación que permite el análisis en profundidad de realidades sociales particulares (Jorrín-Abellán, 2019) y contribuye al estudio de sistemas bien delimitados y en acción (MacDonald \& Walker, 1975), como el nuestro. Existen multitud de aproximaciones a esta tradición de investigación en el área de ciencias sociales. Las más influyentes según Yazan (2016), han sido las propuestas por Robert Yin, Sharan Merriam y Robert Stake. Nosotros nos basaremos en la propuesta de Stake $(2005,2010)$ que determina que un Estudio de Caso debe permitirnos comprender en profundidad y de forma holística, empírica e interpretativa, la persona, innovación o programa que es objeto de estudio. Estas aportaciones nos encaminaron a determinar que el Estudio de Caso era la tradición de investigación cualitativa idónea para abordar el fenómeno seleccionado: las Ecologías de Aprendizaje. Dentro de las tres tipologías de Estudio de Caso que propone Stake (2005), el nuestro se ajusta a la tipología de un Estudio de Caso colectivo, ya que, el interés de la investigación se centra en el estudio de un fenómeno y una población, y se seleccionan para ese fin diversos casos que han de ser estudiados intensa y profundamente.

Así pues, se propone en primer lugar, la definición de los elementos que componen nuestro caso "Docentes Universitarios Excelentes en España". Esto nos sirvió de guía para comprender el transcurso de la investigación, sus objetivos, características y evolución, teniendo en cuenta que el objetivo de investigación se centra en analizar cómo configuran sus EdA los mejores profesores universitarios de los diferentes ámbitos de conocimiento. A este fin fue necesario describir los límites del caso a estudiar y concretar la estructura conceptual del caso que se presenta, atendiendo a la definición de los issues o tensiones de investigación, y de las Declaraciones Temáticas; lo que nos permitió iluminar el funcionamiento del caso (Stake, 2005). Como se explica a continuación, y según este mismo autor, la estructura conceptual de cualquier Estudio de Caso pretende tanto mejorar la comprensión de la naturaleza del caso, como definir sus singularidades y complejidades, proporcionando estructuras que guían la recogida de datos y esquemas para presentar las interpretaciones de los participantes (Stake, 2005). Tomando como referencia las consideraciones de Stake (2005), autores como Jorrín-Abellán (2006) y Villagrá (2012) definen un issue como una tensión entre dos cuestiones, que se expresa, generalmente, de forma interrogativa. Con su determinación dilucidamos cuáles fueron las cuestiones en las que nos debíamos enfocar. Entendiendo y asumiendo, por tanto, que la complejidad que entrañaba nuestro caso era prácticamente inabarcable en su totalidad. En nuestro Estudio de Caso hemos definido dos issues, y son los siguientes:

a) ¿Qué elementos componen la dimensión personal de las Ecologías de Aprendizaje en el proceso de desarrollo profesional de los mejores docentes universitarios?

b)¿Qué elementos componen la dimensión contextual de las Ecologías de Aprendizaje en el proceso de desarrollo profesional de los mejores Docentes Universitarios? 
El siguiente paso fue generar las declaraciones temáticas, que concretan, organizan y especifican lo establecido en los issues, y permiten al investigador anticipar el devenir del caso y seleccionar la información que nos interesa extraer del caso, haciéndolo abarcable para el investigador (Jorrín-Abellán, 2006). La organización de las declaraciones temáticas alrededor de los issues, responde a la necesidad de ir ahondando en el caso, de lo más general a lo más concreto (Jorrín-Abellán, 2006). Así pues, dentro de la primera tensión de investigación o issue (relativa a la dimensión personal de las EdA), se han especificado tres Declaraciones Temáticas (DT):

- DT_1) La configuración de la identidad profesional y su influencia en el desarrollo profesional.

- DT_2) La relevancia de la motivación en el aprendizaje a lo largo y ancho de la vida.

- DT_3) El papel de las concepciones en torno a la profesión docente, a la formación permanente y al desarrollo profesional del docente universitario.

Siguiendo con la segunda tensión de investigación o issue (relativa a la dimensión contextual de las EdA), se han propuesto tres Declaraciones Temáticas:

- DT_4) La relevancia de las experiencias personales en la configuración de las Ecologías de Aprendizaje docente (relaciones, recursos, estrategias, influencias).

- DT_5) La influencia de las experiencias académicas en el desarrollo docente (relaciones, recursos, estrategias, influencias).

- DT_6) Valoración de las experiencias laborales para el desarrollo profesional (relaciones, recursos, estrategias, influencias).

Nuestro Estudio de Caso se centra en el análisis de los "Docentes Universitarios Excelentes en España", así pues, nos situamos en el territorio español y en el ámbito universitario.

Estos dos elementos componen parte de los límites de nuestro caso, pero, además, otro de sus límites es la excelencia docente.

Esta última cualidad que delimita nuestro caso, viene definida por los resultados de la valoración anual derivada del programa de evaluación institucional del profesorado universitario denominado Docentia, que se ha implantado en la mayoría de las universidades españolas.

La principal función del estudio es analizar cómo configuran sus Ecologías de Aprendizaje los docentes universitarios que obtuvieron la calificación de excelente en el programa Docentia. Así, la propiedad de "excelencia docente" define nuestro caso y compone uno de sus límites más claros.

\section{Método}

En este apartado se detalla el estudio empírico desarrollado: fases de la investigación, técnica de recogida de datos, participantes y análisis de datos. Cabe mencionar que este estudio fue llevado a cabo de acuerdo con las recomendaciones del Comité de Ética de la Investigación y la Docencia de la Universidade da Coruña y de la Declaración de Helsinki.

\subsection{Fases de la investigación}

La presente investigación se ha desarrollado a lo largo de cuatro fases: fase preparatoria, trabajo de campo, fase analítica y fase informativa (Rodríguez, Gil, \& García, 1996) (ver Figura 1). Describiremos seguidamente cada una de estas fases y, a continuación, concretamos las acciones específicas desarrolladas en la realización de este estudio. 


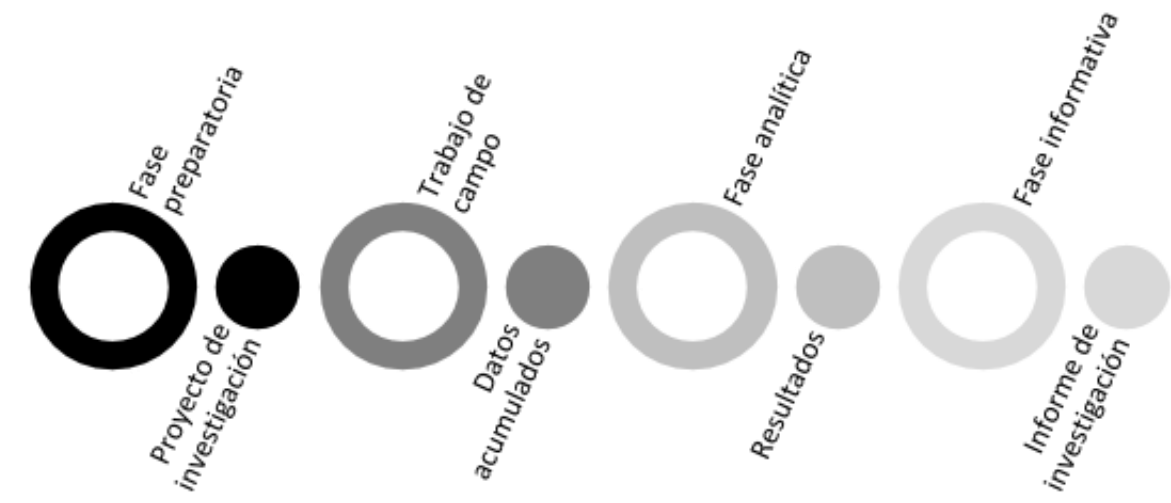

Fig 1. Fases y productos del proceso de investigación cualitativa. Elaboración propia a partir de Rodríguez y colaboradores (1996)

La primera fase, denominada Fase preparatoria, tiene como finalidad el diseño del proyecto de investigación. Algunas de las tareas que se llevan a cabo son: la elección del tema de investigación (pregunta inicial de investigación), la formulación del problema, y la concreción del diseño metodológico de la investigación.

La Fase de trabajo de campo tiene como objetivo la recolección de la información. Así, las tareas fundamentales son: la captación de las unidades y acceso al campo, y la obtención de los datos. Tal y como se explicará posteriormente, en este estudio se ha empleado la entrevista como técnica de recogida de datos.

En tercer lugar, la Fase analítica conlleva la obtención de los resultados. Así, las principales tareas que se llevan a cabo son: el tratamiento de los datos (procesamiento y organización de la información), y la interpretación de los resultados.

Finalmente, durante la Fase informativa tiene lugar la presentación de los resultados y la elaboración de las conclusiones. Esta fase concluye con la presentación del informe de investigación.

\subsection{Técnica de recogida de datos}

La técnica de recogida de datos empleada ha sido la entrevista en profundidad (Creswell \& Creswell, 2018), puesto que permite "explorar la forma en que los sujetos experimentan y entienden su mundo" (Kvale, 2011, p.30). Teniendo en cuenta la clasificación de los diferentes tipos de entrevista propuesta por Verd y Lozares (2016) (ver Figura 2), se puede afirmar que las entrevistas desarrolladas son: a) individuales, es decir se han llevado a cabo de forma independiente con cada informante ; b) simultáneas en el tiempo y en el espacio, ya que se han realizado presencialmente, c) semidirigidas, puesto que para asegurarse de que se abordaban los temas claves con todos los participantes, se ha elaborado un guion con diferentes preguntas; $y$ d) temáticas, debido a que se establecieron con antelación los asuntos a tratar. Concretamente, se han realizado dos entrevistas con distintas temáticas y en momentos temporales diferentes a cada participante; una primera entrevista, de tipo biográfico, y una segunda entrevista, sobre mecanismos de aprendizaje y actualización profesional.

- A través de la primera entrevista, se recogieron los datos personales de cada docente, así como información acerca de su trayectoria profesional y sus expectativas de formación. Se trata de comprender la historia de vida de cada docente (Bolívar, Domingo, \& Fernández, 2001). El objetivo fundamental consiste en situar el propio relato del profesor o profesora en el marco de un análisis contextual más amplio, es decir, construir una narrativa de la acción dentro de una teoría del contexto (Goodson, 2004). 
- En segundo término, la entrevista sobre mecanismos de aprendizaje y actualización profesional, abarcó todo lo relacionado con los recursos y procesos de aprendizaje que los docentes ponen en juego para contribuir a su formación permanente. Es decir, se trata de conocer y reflexionar sobre los entornos y ambientes en los que interactúan y aprenden, además de comprender el sentido y uso que les adjudican a las tecnologías digitales para la mejora de su práctica docente. Todo ello, contribuye a identificar específicamente las ecologías de aprendizaje de cada uno de los participantes.

\begin{tabular}{|c|}
\hline $\begin{array}{c}\text { Grado de } \\
\text { estandarización }\end{array}$ \\
\hline Entrevista \\
dirigida o \\
estructurada \\
Entrevista \\
semidirigida o \\
semiestructurada \\
Entrevista no \\
dirigida o no \\
estructurada \\
\end{tabular}

\begin{tabular}{|c|}
\hline Contenido \\
\hline $\begin{array}{c}\text { Entrevista } \\
\text { temática } \\
\text { Entrevista } \\
\text { genérica }\end{array}$ \\
\hline
\end{tabular}

\begin{tabular}{|c|c|}
\hline $\begin{array}{c}\text { Grado de } \\
\text { simultaneidad }\end{array}$ & $\begin{array}{c}\text { Número de } \\
\text { participantes } \\
\text { simultaneas en el } \\
\text { tiempo y en el } \\
\text { espacio } \\
\text { Entrevistas } \\
\text { simultaneas en el } \\
\text { tiempo, pero no } \\
\text { en el espacio } \\
\text { Entrevista sin } \\
\text { simultaneidad en } \\
\text { el tiempo ni en el } \\
\text { espacio }\end{array}$ \\
\\
\end{tabular}

Fig. 2. Clasificación de los diferentes tipos de entrevistas. Elaboración propia a partir de Verd y Lozares (2016)

Una vez llegados a este punto, es preciso destacar que el proceso de diseño y realización de las entrevistas ha tenido lugar en tres momentos: preparación, desarrollo y valoración (Massot, Dorio, \& Sabariego, 2009). En la Figura 3, se detallan las tareas llevadas a cabo en cada uno de esos momentos.

En relación a la fase de preparación, cabe destacar que los dos guiones de entrevista fueron elaborados en base a la literatura sobre Ecologías de Aprendizaje, nuevos modelos de formación y desarrollo profesional docente. Estos guiones iniciales fueron sometidos a un juicio de tres expertos en la línea de investigación de Ecologías de Aprendizaje y desarrollo profesional, cuyas recomendaciones y sugerencias fueron tomadas en cuenta para elaborar el guion definitivo que se utilizó para realizar las entrevistas.

Con respecto a la fase de desarrollo es preciso mencionar que cada entrevista se realizó en una única sesión, lo que da lugar a dos sesiones (en diferentes momentos temporales) por informante. La duración aproximada de cada sesión fue de entre 45 y 90 minutos, dependiendo tanto de la temática como del participante. Las entrevistas fueron realizadas presencialmente por los miembros del equipo del proyecto de cada Universidad en el lugar acordado con cada docente entrevistado, que en la mayoría de las ocasiones era el despacho propio del informante. 


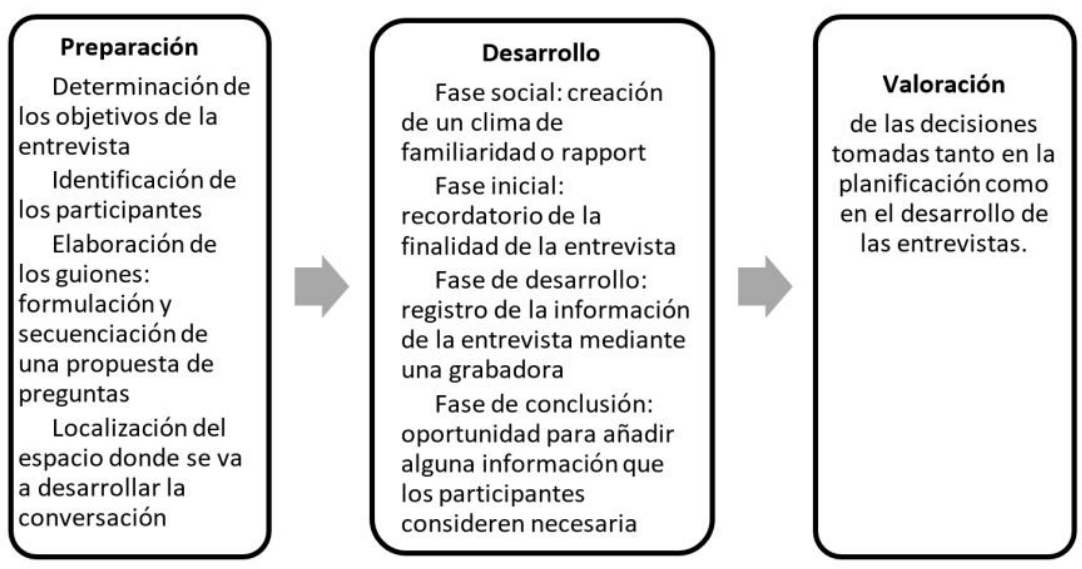

Fig. 3. Proceso de diseño y realización de las entrevistas. Elaboración propia.

\subsection{Participantes}

Para hacer la selección de los informantes, atendiendo al criterio de excelencia, nos basamos en los resultados de las evaluaciones institucionales anuales que proporciona el Programa Docentia en las diferentes universidades. El Programa de Apoyo a la Evaluación de la Actividad Docente del Profesorado Universitario se puso en marcha en 2007 por la Agencia Nacional de la Evaluación de la Calidad y Acreditación (ANECA), en estrecha coordinación con las Agencias de Evaluación Autonómicas Este tiene el objetivo de apoyar a las universidades en el diseño de mecanismos propios para gestionar la calidad de la actividad docente del profesorado universitario y favorecer su desarrollo y reconocimiento.

En total contamos con 72 informantes pertenecientes a las seis universidades que colaboran en el proyecto de investigación en el que se enmarca esta investigación (ECO4LEARN-HE) y que se ubican dentro del territorio nacional: Universidade da Coruña (UDC), Universitat Oberta de Catalunya (UOC), Universidad Politécnica de Madrid (UPM), Universidad de Navarra (UNAV), Universidad de Alicante (UA) y Universidad de Granada (UGR). Estos informantes fueron seleccionados por obtener resultados excelentes en el programa Docentia. Este aspecto constituyó uno de los criterios de inclusión y, además, una de las cualidades que definen los límites de nuestro caso. Dado que esta información es confidencial, y teniendo en cuenta las recomendaciones del Comité de Ética, fue el rectorado de cada universidad el encargado de ponerse en contacto con los docentes y solicitar su participación en el estudio. Una vez el profesorado confirmó su colaboración, nos fueron proporcionados sus datos de contacto. En la Tabla 1, que mostramos a continuación, se presentan los participantes según su universidad, el área de conocimiento a la que pertenecen y su sexo.

Tabla 1. Datos sobre los participantes

\begin{tabular}{|c|c|c|c|c|c|c|c|}
\hline $\begin{array}{c}\text { Rama de } \\
\text { conocimiento }\end{array}$ & Ciencias & $\begin{array}{l}\text { CC de la } \\
\text { Salud }\end{array}$ & $\begin{array}{c}\text { Artes y } \\
\text { Humanidade } \\
s\end{array}$ & Social-jurídico & $\begin{array}{l}\text { Ingeniería-Arq } \\
\text { uitectura }\end{array}$ & \multicolumn{2}{|c|}{$\begin{array}{l}\text { SUBTOTAL } \\
\text { POR UNIVERS. }\end{array}$} \\
\hline UDC & $\begin{array}{l}\text { Hombre: } 1 \\
\text { Mujer: } 1\end{array}$ & $\begin{array}{l}\text { Hombre: } 3 \\
\text { Mujer: } 2\end{array}$ & $\begin{array}{l}\text { Hombre: } 2 \\
\text { Mujer: } 2\end{array}$ & $\begin{array}{l}\text { Hombre: } 4 \\
\text { Mujer: } 2\end{array}$ & $\begin{array}{l}\text { Hombre: } 2 \\
\text { Mujer: } 3\end{array}$ & $\begin{array}{l}H: 12 \\
M: 10\end{array}$ & 22 \\
\hline UOC & $\begin{array}{l}\text { Hombre: } 1 \\
\text { Mujer: } 1\end{array}$ & $\begin{array}{l}\text { Hombre: } 1 \\
\text { Mujer: } 1\end{array}$ & $\begin{array}{l}\text { Hombre: } 2 \\
\text { Mujer: } 2\end{array}$ & $\begin{array}{l}\text { Hombre: } 1 \\
\text { Mujer: } 1\end{array}$ & $\begin{array}{l}\text { Hombre: } 1 \\
\text { Mujer: } 1\end{array}$ & $\begin{array}{l}H: 6 \\
M: 6\end{array}$ & 12 \\
\hline UPM & $\begin{array}{l}\text { Hombre: } 1 \\
\text { Mujer: } 1\end{array}$ & & & & $\begin{array}{l}\text { Hombre: } 6 \\
\text { Mujer: } 2\end{array}$ & $\begin{array}{l}\mathrm{H}: 7 \\
\mathrm{M}: 3\end{array}$ & 10 \\
\hline UNAV & $\begin{array}{l}\text { Hombre: } 1 \\
\text { Mujer: } 1\end{array}$ & & $\begin{array}{l}\text { Hombre: } 1 \\
\text { Mujer: } 0\end{array}$ & $\begin{array}{l}\text { Hombre: } 1 \\
\text { Mujer: } 0\end{array}$ & & $\begin{array}{l}H: 3 \\
M: 1\end{array}$ & 4 \\
\hline UA & $\begin{array}{l}\text { Hombre: } 1 \\
\text { Mujer: } 0\end{array}$ & $\begin{array}{l}\text { Hombre: } 0 \\
\text { Mujer: } 3\end{array}$ & $\begin{array}{l}\text { Hombre: } 1 \\
\text { Mujer: } 0\end{array}$ & $\begin{array}{l}\text { Hombre: } 1 \\
\text { Mujer: } 1\end{array}$ & $\begin{array}{l}\text { Hombre: } 1 \\
\text { Mujer: } 0\end{array}$ & $\begin{array}{l}\mathrm{H}: 4 \\
\mathrm{M}: 4\end{array}$ & 8 \\
\hline UGR & $\begin{array}{l}\text { Hombre: } 3 \\
\text { Mujer: } 1\end{array}$ & $\begin{array}{l}\text { Hombre: } 2 \\
\text { Mujer: } 1\end{array}$ & $\begin{array}{l}\text { Hombre: } 2 \\
\text { Mujer: } 2\end{array}$ & $\begin{array}{l}\text { Hombre: } 3 \\
\text { Mujer: } 1\end{array}$ & $\begin{array}{l}\text { Hombre: } 1 \\
\text { Mujer: } 0\end{array}$ & $\begin{array}{l}\mathrm{H}: 11 \\
\mathrm{M}: 5\end{array}$ & 16 \\
\hline $\begin{array}{c}\text { SUBTOTAL } \\
\text { POR RAMAS }\end{array}$ & 26 & 26 & 28 & 30 & 34 & partic & ntes \\
\hline
\end{tabular}




\subsection{Análisis de datos}

Desde una perspectiva cualitativa de investigación, el análisis de datos se basa en un proceso de interpretación, asignación de significados y categorización de la información recogida. El objetivo del análisis de las entrevistas era conocer en profundidad todos los elementos que conforman las EdA de los docentes entrevistados: su historia de vida, su contexto profesional y personal, su formación desde sus inicios en la profesión y actualmente, sus prácticas docentes, sus creencias con respecto a la autoformación, la formación permanente, las TIC, sus mecanismos de actualización, etc. Algunos autores, como Schoenfeld (1999), afirman que son estos aspectos los que moderan las prácticas docentes de los profesores. Por tanto, asumiendo que los informantes de nuestro caso son excelentes docentes universitarios, podremos entender qué factores influyen y definen la excelencia docente y por ende la calidad de la enseñanza universitaria.

Una de las características de la investigación cualitativa es que se suele acumular gran cantidad de información a analizar. Miles y Huberman (1984) presentan un modelo interactivo de análisis que ayuda a abordar el conjunto de los datos cualitativos de naturaleza textual. Este modelo presenta tres pasos recurrentes que se han combinado en la interpretación de la información recolectada: la reducción de datos, la representación de los datos y la elaboración de conclusiones y su verificación. Estas tareas u operaciones constituyen el proceso analítico básico, común a la mayoría de los estudios en los que se trabaja con datos cualitativos.

Este proceso sistemático de análisis puede seguir una secuencia inductiva o deductiva (Goetz \& LeCompte, 1988). Miles y Huberman (1984) establecen que usualmente ambas estrategias se combinan y no son excluyentes, y este ha sido el doble proceso que hemos seguido en esta investigación.

En primer lugar, y para proceder de manera analítica al encuentro de significados emergidos de los datos ya recogidos y transcritos, utilizamos la codificación mixta, donde se combinan estrategias inductivas y deductivas.

Nuestra aproximación parte de la creación de un sistema de categorías de análisis basado en la teoría acerca de las Ecologías de Aprendizaje y de las tensiones de investigación propuestas en nuestro estudio, pero siempre en busca de sustento en el material empírico recolectado.

Este manual de códigos no era cerrado ni estático y estaba abierto a nuevas interpretaciones y descubrimientos, por lo que fue sufriendo cambios a medida que se codificaba y se releía el material. Se crearon categorías y subcategorías, se unieron categorías, se eliminaron otras, etc. Se produjeron varias rondas de codificación combinando estrategias de codificación abierta, axial y selectiva. Este proceso se llevó a cabo con la ayuda del Software Atlas.ti (Versión 7) y con la participación de cuatro investigadoras de la Universidade da Coruña.

Para proceder con los movimientos analíticos en el corpus de datos, cabe destacar que las investigadoras acordaron el sistema y estrategias de codificación previamente. A medida que se sucedían las rondas de codificación, se incrementaba la densidad de las categorías generadas. Finalmente, después de alcanzada la saturación teórica de las diferentes categorías, se obtuvo un sistema de códigos que vinculaba todos los fragmentos de los casos entrevistados sobre los diferentes tópicos, pero que también conectaba las nociones teóricas con las ideas que aparecieron en los datos (Borda et al., 2017). El esquema definitivo se presenta en la siguiente Figura 4. 


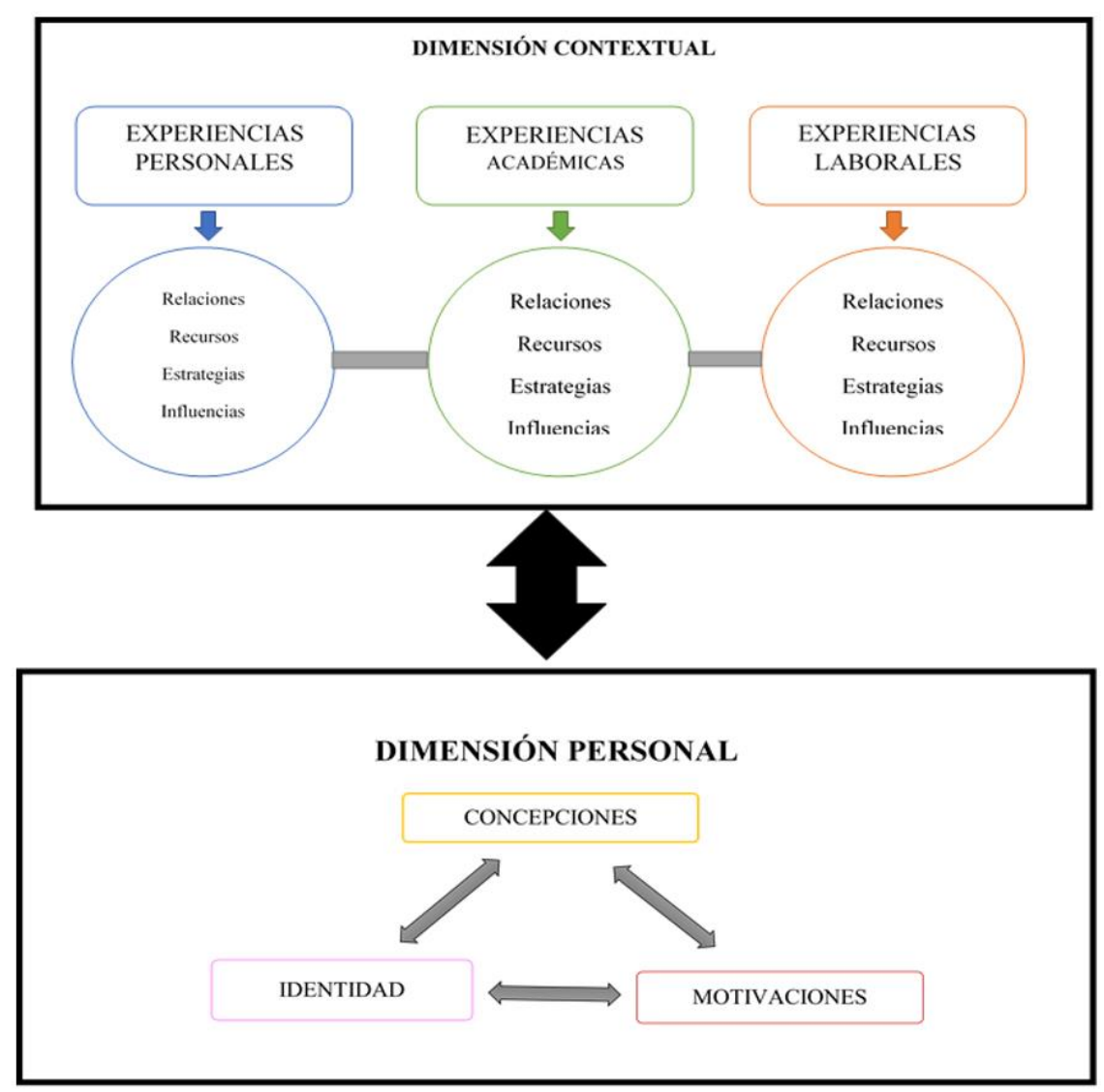

Fig. 4. Sistema de categorías emanado del Estudio de Caso

\section{Guidelines para estudios siguientes}

A través del análisis de las EdA se podrían identificar patrones de comportamiento para propiciar y alentar el aprendizaje y el desarrollo profesional de los trabajadores de cualquier ámbito ocupacional, aprovechando las oportunidades que ofrecen los sistemas formativos formales, no formales e informales y, de esta forma, poner en marcha mecanismos y procesos que favorezcan el aprendizaje a lo largo de la vida.

Desde la óptica del enfoque de investigación elegido, cabe destacar que la investigación cualitativa constituye la metodología más acertada para el estudio de las EdA, y de cualquier otro fenómeno complejo -por cuanto permite el abordaje de la amplitud de aspectos que comporta su estudio-; poliédrico -por cuanto se pueden desvelar las diversas implicaciones que requiere su análisis- y emergente -ya que los aspectos desconocidos serán más factibles de abordar desde la perspectiva comprensiva que comporta la investigación cualitativa-. Únicamente desde una indagación cualitativa se puede realizar un acercamiento a la realidad de cada persona y de cada grupo social para que desde sus percepciones, experiencias y comportamientos se puedan desvelar los procesos, mecanismos y componentes de su aprendizaje y su desarrollo profesional.

\section{Aplicabilidad en otros contextos}

El análisis de las EdA y su influencia en el desarrollo personal y profesional es aplicable y transferible a todos y cada uno de los contextos en los que habitan y por los que transitan las personas. 
Así pues, el conocimiento del significado del constructo EdA es de gran utilidad para el análisis de los procesos de aprendizaje de cualquier persona y, en particular, de cualquier profesional.

El presente trabajo permitirá a otros investigadores conocer el significado del constructo EdA y de la línea de investigación que se está desarrollando de manera que puedan aplicarlo en otros estudios y en el análisis de los procesos de aprendizaje de cualquier persona y cualquier profesional en la realidad actual.

Finalmente, tanto la tradición de investigación del Estudio de Caso como la técnica de la entrevista pueden ser empleadas en investigaciones que estudien fenómenos complejos, que analicen problemas estrechamente vinculados al contexto en el que se desarrollan y que requieran la obtención de información más completa, holística y profunda.

\section{Conclusiones}

En lo que respecta al primer objetivo (analizar cómo los mejores docentes universitarios españoles configuran sus Ecologías de Aprendizaje y las utilizan en beneficio de su desarrollo profesional) y específicamente, en relación a los resultados generados en torno a los dos issues planteados, es preciso destacar que el análisis de los datos obtenidos ha permitido poner de manifiesto cómo cada docente construye su sistema de aprendizaje y articula sus formatos de desarrollo profesional.

Así pues, dentro de los factores de carácter contextual emergidos, se ha constatado la importancia de la tecnología en el quehacer docente, así como, en el acceso a las diversas oportunidades de formación en los diferentes contextos formales, no formales e informales.

Del mismo modo, es preciso destacar la importancia de las relaciones entre colegas, como mecanismos altamente eficaces para promover el aprendizaje profesional docente.

Esto coincide con los resultados de estudios como los de Jarauta (2013), Mauri, Clarà y Colomina (2013) o Martín-Gutiérrez, Conde-Jiménez y Mayor-Ruiz (2014), donde se determina la importancia que tienen esos elementos en el proceso de aprendizaje docente. Asimismo, dentro de la esfera personal, se destacan la motivación hacia la mejora del desempeño docente, la identidad profesional - generada a partir de las experiencias vitales, académicas y laborales -, y las valoraciones y concepciones sobre el desarrollo profesional y la formación continua, como factores determinantes en la configuración de las EdA de los mejores docentes universitarios. Este hallazgo coincide con lo aportado por González-Sanmamed, Muñoz-Carril y Santos-Caamaño (2019) en un reciente estudio.

En cuanto al segundo objetivo, los resultados obtenidos han constatado la pertinencia del Estudio de Caso como tradición de investigación para el análisis del desarrollo profesional docente desde la perspectiva ecológica.

El manejo del constructo ha puesto de manifiesto que la naturaleza compleja, multidimensional y entramada de las EdA, así como su estado de desarrollo embrionario, hacen que la metodología cualitativa sea la más apropiada para su estudio.

Nuestro objetivo era retratar esta realidad de una forma holística y profunda, y dado que los límites del fenómeno estaban esencialmente definidos, el Estudio de Caso se presentó como la tradición idónea para el desarrollo de la investigación.

Sin embargo, también se considera para futuros estudios un enfoque de investigación de carácter mixto, donde una aproximación cuantitativa del estudio de los elementos que componen las EdA, complementen el estudio cualitativo llevado a cabo. 


\section{Referencias}

Arantzamendi, M., López-Dicastillo, O., \& García-Vivar, C. (2012). Investigación cualitativa. Pamplona: Ediciones Eunate.

Bolívar, A., Domingo, J., \& Fernández, M. (2001). La Investigación biográfico-narrativa en educación. Enfoque y metodología. Madrid. La Muralla

Borda, P., Dabenigno, V., Freidin, B, \& Güelman, M. (2017). Estrategias para el análisis de datos cualitativos. Instituto de Investigaciones Gino Germani. Recuperado de http://209.177.156.169/libreria_cm/archivos/pdf_1605.pdf

Creswell, J. W., \& Creswell, J. D. (2018). Research Design: Qualitative, Quantitative \& Mixed Methods Approaches. London: Sage Publications.

Duart, J. M., \& Mengual-Andrés, S. (2014). Impacto de la sociedad del conocimiento en la universidad y en la comunicación científica. RELIEVE. Revista Electrónica de Investigación y Evaluación Educativa, 20(2). https://doi.org/10.7203/relieve.20.2.4343

Fernández Enguita, M. (2016). Se non è ver, è ben trovato. Las leyendas urbanas del profesorado y una posible explicación de las mismas. In J. M. Valle and J. Manso (Eds.), La cuestión docente a debate. Nuevas perspectivas (pp. 99-110). Madrid: Narcea.

Goetz, J., \& Lecompte, M. (1988). Etnografía y diseño cualitativo en investigación cualitativa. Madrid, España: Morata.

González-Sanmamed, M., Estévez, I., Souto-Seijo, A., \& Muñoz-Carril, P. C. (2020). Digital learning ecologies and professional development of university professors. Comunicar, 28(62), 9-18. https://doi.org/10.3916/C62-2020-01

González-Sanmamed, M., Muñoz-Carril, P. C., \& Santos-Caamaño, F. J. (2019). Key components of learning ecologies: A Delphi assessment. British Journal of Educational Technology, 5(4), 1639-1655. https://doi.org/10.1111/bjet.12805

González-Sanmamed, M., Sangrà, A., Souto-Seijo, A., \& Estévez, I. (2018). Ecologías de aprendizaje en la Era Digital: desafíos para la Educación Superior. Publicaciones, 48(1), 2545. https://doi.org/10.30827/publicaciones.v48i1.7329

González-Sanmamed, M., Souto-Seijo, A., González, I., \& Estévez, I. (2019). Aprendizaje informal y desarrollo profesional: análisis de las ecologías de aprendizaje del profesorado de educación infantil. Edutec. Revista Electrónica de Tecnología Educativa, (68), 70-81. https://doi.org/10.21556/edutec.2019.68.1305

Goodson, I. (2004). Historias de vida del profesorado. Barcelona: Octaedro.

Jarauta, B. (2013). Saberes pedagógicos para una didáctica reflexiva. In J. L. Medina, B. Jarauta, F. Imbernón, and A. Bartolomé (Eds.), Enseñanza y aprendizaje en la educación superior (pp. 85-122). Madrid: Síntesis.

Jorrín-Abellán, I. M. (2006). Perfil formativo generado en los entornos CSCL. Un estudio de caso (tesis doctoral). Universidad de Valladolid, Valladolid, España.

Jorrín-Abellán, I. M. (2019). Hopscotch 2.0: an enhanced version of the Model for the Generation of Research Designs in Social Sciences and Education. Georgia Educational Researcher, 16(1). https://doi.org/10.20429/ger.2019.160103

Kvale, S. (2011). Las entrevistas en Investigación Cualitativa. Madrid: Ediciones Morata.

Martín-Gutiérrez, Á., Conde-Jiménez, J., \& Mayor-Ruiz, C. (2014). La identidad profesional docente del profesorado novel universitario. REDU. Revista de Docencia Universitaria, 12(4), 141160. Recuperado de https://bit.ly/33CWlcC

Massot, I., Dorio, I., \& Sabariego, M. (2009). Estrategias de recogida y análisis de la información. En R. Bisquerra (Ed.), Metodología de la investigación educativa (pp. 329-366). Madrid: La Muralla.

Mauri, T., Clarà, M., \& Colomina, A. G. Y. R. (2013). La contribución al aprendizaje en el lugar de trabajo de los equipos docentes universitarios. un estudio exploratorio. Infancia $y$ Aprendizaje, 36(3), 341-360. https://doi.org/10.1174/021037013807533025 
Miles, M., \& Huberman, A. (1984). Análisis de datos cualitativos. Un libro de consulta de nuevos métodos. NY: Sage Publications.

Pedraz, A., Zarco, J., Ramasco, M., \& Palmar, A.M. (2014). Investigación cualitativa. Madrid: Elsevier.

Rodríguez, G., Gil, J., \& García, E. (1996). Metodología de la investigación cualitativa. Granada: Ediciones Aljibe.

Sangrà, A., González-Sanmamed, M., \& Guitert, M. (2013). Learning Ecologies: Informal professional development opportunities for teachers. Proceedings of the IEEE, 1/201. https://doi.org/10.1109/CICEM.2013.6820171

Sangrá, A., Raffaghelli, J. E., \& Guitert-Catasús, M. (2019). Learning ecologies through a lens: Ontological, methodological and applicative issues. A systematic review of the literature. British Journal of Educational Technology, bjet.12795. https://doi.org/10.1111/bjet.12795

Schoenfeld, A. H. (1999). Models of the teaching process. The Journal of Mathematical Behavior, 18(3), 243-261. https://doi.org/10.1016/S0732-3123(99)00031-0

Stake, R. E. (2005). Qualitative case studies. In N. K. Denzin and Y. S. Lincoln (Eds.), The Sage handbook of qualitative research (3rd. Ed.) (pp. 443-466). Sage.

Stake, R. E. (2010). Qualitative Research: Studying how things work. Nueva York, NY: The Guilford Press.

Verd, J. M., \& Lozares, C. (2016). Introducción a la investigación cualitativa. Fases, métodos y técnicas. Madrid: Síntesis

\section{Agradecimientos}

Este estudio se ha elaborado en el marco del proyecto de investigación ECO4LEARN-HE, financiado por el Ministerio de Economía y Finanzas (Referencia EDU2015-67907-R), y gracias a la financiación recibida por una de las autoras de esta investigación, Iris Estévez, en el programa FPI del Ministerio de Economía, Industria y Competitividad (BES-2016-077330).

\section{Notas Biograficas}

Iris Estévez. Doctora por la Universidade da Coruña y contratada postdoctoral FPI en el Departamento de Pedagogía y Didáctica de la Facultad de Ciencias de la Educación de la misma universidad. Su tesis doctoral se denomina "Ecologías de Aprendizaje de los docentes universitarios de Ciencias de la Salud". Ha realizado dos estancias de investigación internacionales, la última de ellas en la Kennesaw State University (USA). Además, es miembro de Equipo de Investigación Educativa (EIRA) de la Universidade da Coruña

Alba Souto Seijo. Doctora por la Universidade da Coruña y profesora en la Facultad de Educación de la Universidad Internacional de la Rioja. Su tesis doctoral se centra en el estudio de las Ecologías de Aprendizaje de los docentes universitarios de la rama de conocimiento de Ingeniería y Arquitectura. Ha realizado una estancia de investigación en el Institute of Learning \& Teaching in Higher Education (University of Northampton, Reino Unido). Asimismo, es miembro del Equipo de Investigación Educativa (EIRA) de la Universidade da Coruña.

Mercedes González-Sanmamed. Profesora Catedrática de la Universidade da Coruña (España). Tiene concedidos 4 tramos de investigación por la CNEAI. Dirige el grupo de investigación EIRA. Su área de investigación se concentra en la formación de profesorado, la integración de las tecnologías en la educación, y la innovación y la mejora educativa. En estos campos ha participado en 40 proyectos competitivos a nivel internacional, nacional y autonómico, de los cuales en 9 de ellos ha sido IP y en 7 coordinadora. Ha dirigido 15 tesis doctorales. Es autora de más de doscientas publicaciones, ha participado en numerosas conferencias y ponencias en congresos científicos. 